\title{
Preface of the Special Issue in Honor of Professor Jayanta Kumar Ghosh
}

\author{
Subhashis Ghosal \\ North Carolina State University, Raleigh, USA
}

Professor Jayanta Kumar Ghosh, a highly respected mathematical statistician and one of the most well-known Indian statisticians, passed away recently on September 30, 2017, at West Lafayette, Indiana, at the age of eighty, after an illustrious career spanning over six decades. Professor Ghosh immensely contributed to many areas of statistics including sequential analysis, foundations of statistics, higher-order asymptotics, objective Bayesian inference, semiparametric statistics, Bayesian asymptotics, Bayesian nonparametrics, model selection, and high dimensional statistics, as well as applied work in reliability theory, statistical quality control, modeling hydrocarbon discoveries, geological mapping, and DNA fingerprinting. He collaborated with a large number of students and colleagues at the Indian Statistical Institute and Purdue University, and leading researchers in the world, and authored or co-authored about 140 papers, 3 monographs and several edited volumes. Professor Ghosh received numerous awards and honors including the S. S. Bhatnagar Award (1981) for Science and Technology, Jawaharlal Nehru Professor (1996-2002) and Padma Shree (2014) from the Government of India, the Mahalanobis Gold Medal of the Indian Science Congress (1998), the P. V. Sukhatme Prize (2000), the International Indian Statistical Association Lifetime Achievement Award (2009), Fellows of the Institute of Mathematical Statistics (1990), the Indian National Science Academy, and the International Society for Bayesian Analysis (2012), Doctor of Science from the Indian Statistical Institute (2012). He also served as Presidents of the International Statistical Institute (1993-1995) and Statistics Section of the Indian Science Congress (1991), and as the editor of Sankhyā (19881999). To celebrate his life and to remember his exceptional contributions, Sankhya is pleased to bring in this special volume in the honor of Professor Jayanta Kumar Ghosh. 
The present special issue of Sankhyā contains nine papers from prominent researchers, who were close to Professor Ghosh, worked in areas where Professor Ghosh made very important contributions, or are leading researchers from contemporary topics Professor Ghosh was interested in. The article "M-estimation in multistage sampling procedures" by Atul Mallik, Moulinath Banerjee and George Michailidis provides a unified asymptotic theory of convergence for two-stage M-estimators that apply to change-point estimation, inverse isotonic regression, classification, mode and cusp estimation, showing that convergence rates of M-estimators can be accelerated by collecting samples in more than one stages. The paper "Frequentist properties of Bayesian multiplicity control for multiple testing of normal means" by Sean Chang and James O. Berger establishes controls over multiple testing characteristics of Bayesian tests for several normal means. The review paper "Density prediction and the Stein phenomenon" by Malay Ghosh, Tatsuya Kubokawa and Gauri Sankar Datta outlines the recent developments on Stein's shrinkage phenomenon for predictive density estimation in terms of several divergence measures. Qifan Song and Guang Cheng obtain posterior consistency for estimating a long vector with an unknown blocking structure using a fused t-prior in their article "Bayesian fusion estimation via t-shrinkage". The paper "Adaptive Bayesian credible bands in regression with a Gaussian process prior" by Suzanne Sniekers and Aad van der Vaart investigates frequentist coverage of Bayesian credible sets for nonparametric regression with unknown smoothness. A unifying framework of several popularly used priors such as horseshoe for sparse estimation is considered in the paper "Global-local mixtures: a unifying framework" by Anindya Bhadra, Jyotishka Datta, Nicholas G. Polson and Brandon T. Willard. The article "Posterior contraction rates for stochastic block models" by Prasenjit Ghosh, Debdeep Pati and Anirban Bhattacharya investigates posterior contraction properties in a stochastic block model for common priors such as multinomial-Dirichlet priors on cluster indicators and beta priors on the probabilities of the random edge indicators. Ryan Martin and Bo Ning in their paper "Empirical priors and coverage of posterior credible sets in a sparse normal mean model" obtain coverage of an empirical Bayesian credible set for a normal mean vector. For sparse high-dimensional linear models, a relatively new estimator called SLOPE is shown to possess good multiple testing abilities in the article "On the asymptotic properties of SLOPE" by Michal Kos and Malgorzata Bogdan. We gratefully acknowledge the contributions of all these authors for promptly accepting our invitation to 
contribute papers, submit on schedule and for the high quality of their papers. Without their enthusiastic response, the project of this special volume would not have succeeded.

We are indebted to a number of people. Professor Dipak Dey, Editor-inChief of Sankhyā, motivated us to edit a special issue in honor of Professor Ghosh. The editorial staff of Sankhyā, Sarvagnan Subramnian set up the system for the special issue and helped with technical problems in numerous occasions, which allowed for a smooth submission and editorial process. Several anonymous referees worked tirelessly on multiple rounds of vigorous reviews within a limited time, which made it possible to publish the special issue on schedule. Unfortunately, due to confidentiality reasons, we cannot publish their names here and personally thank them, but we express our sincere gratitude. Finally, I would like to thank my co-editors, Professors Tapas Samanta and Surya T. Tokdar, for their tireless work and time they invested to make this special issue a success.

Subhashis Ghosal

Chief guest editor

Publisher's Note. Springer Nature remains neutral with regard to jurisdictional claims in published maps and institutional affiliations.

Subhashis GHosal

North Carolina State University,

RALEIGH, NC, USA

E-mail: sghosal@ncsu.edu 